\title{
Suspicious for Malignancy
}

National Cancer Institute

\section{Source}

National Cancer Institute. Suspicious for Malignancy. NCI Thesaurus. Code C121869.

A microscopic finding indicating the presence of a cellular infiltrate or isolated cells that have features suspicious for malignancy. 